\title{
UTILIZAÇÃO DA ESCALA DE AVALIAÇÃO DA ESPIRITUALIDADE EM PACIENTES PORTADORES DE LESÃO RENAL EM HEMODIÁLISE
}

\author{
Ana Lúcia Rodrigues Correia¹, Islene Victor Barbosa², Francisca Elisângela Teixeira Lima ${ }^{3}$, Virna Ribeiro Feitosa Cestari ${ }^{4}$, Rita
} Mônica Borges Studart ${ }^{2}$, Francisca Lígia de Medeiros Martins ${ }^{5}$

${ }^{1}$ Enfermeira. Especialista em Enfermagem em Nefrologia. Universidade Estadual do Ceará. Fortaleza, CE, Brasil.

${ }^{2}$ Enfermeira. Doutora em Enfermagem. Docente do Departamento de Enfermagem da Universidade de Fortaleza. Fortaleza, CE, Brasil.

${ }^{3}$ Enfermeira. Doutora em Enfermagem. Docente do Departamento de Enfermagem da Universidade Federal do Ceará. Fortaleza, CE, Brasil.

${ }^{4}$ Discente do curso de graduação em Enfermagem. Universidade de Fortaleza. Fortaleza, CE, Brasil.

${ }^{5}$ Enfermeira. Mestre em Enfermagem. Docente do Departamento de Enfermagem da Universidade de Fortaleza. Fortaleza, CE, Brasil.

RESUMO: Objetivou-se avaliar a espiritualidade de pacientes em hemodiálise, fundamentada na Escala de Avaliação da Espiritualidade em Contextos de Saúde. Estudo descritivo e transversal, realizado com 80 pacientes internados por lesão renal em hemodiálise. Dados coletados de dezembro de 2011 a janeiro de 2012, por meio de uma escala autoaplicável de avaliação da espiritualidade. Dos 80 pacientes, 93,75\% possuíam alguma crença religiosa. Quanto às expressões de espiritualidade, $57,5 \%$ estavam de acordo com a variável "As minhas crenças espirituais/religiosas dão sentido à minha vida"; $70 \%$, com "Minha fé e crença dão-me força nos momentos difíceis"; 48,8\%, com "Vejo o futuro com esperança"; 21,3\%, "Sinto que minha vida mudou para melhor"; e 63,7\%, com "Aprendi a dar valor às pequenas coisas da vida". Constatou-se que o espiritual/religioso exerce grande influência na saúde do paciente com lesão renal em hemodiálise, evidenciando a importância de profissionais preparados para lidarem com esta temática.

DESCRITORES: Espiritualidade; Insuficiência renal crônica; Diálise renal; Enfermagem.

\section{USE OF A SPIRITUALTTY SELF-RATING SCALE IN PATIENTS WITH KIDNEY DISEASE UNDERGOING HEMODIALYSIS}

ABSTRACT: The present study aimed to assess the spiritual well-being of patients undergoing hemodialysis, based on a Spirituality Self-Rating Scale for use in health care. Crosssectional descriptive study with 80 patients hospitalized to treat kidney disease undergoing hemodialysis. Data collected from December 2011 to January 2012, through a self-rating scale for assessment of spirituality. Of the 80 patients, $93.75 \%$ had some religious belief. Regarding the expressions of spirituality, $57.5 \%$ agreed with the expression "My spiritual/religious beliefs give a meaning to my life"; $70 \%$, with the expression "My faith and belief give me strength at difficult times"; $48.8 \%$, with "I have hope in the future"; $21.3 \%$ with "I feel my life has changed for the better"; and $63.7 \%$, with "I learned to appreciate the little things in life". It has been observed that the spiritual/religious aspect has great influence on the health status of patients with kidney disease undergoing hemodialysis, highlighting the importance for health professionals to be prepared for dealing with this issue.

DESCRIPTORS: Spirituality; Chronic kidney Disease; Renal Dialysis; Nursing.

\section{UTILIZACIÓN DE LA ESCALA DE EVALUACIÓN DE LA ESPIRITUALIDAD EN PACIENTES CON LESIÓN RENAL EN HEMODIÁLISIS}

RESUMEN: Se objetivó evaluar la espiritualidad de pacientes en hemodiálisis, fundamentada en la Escala de Evaluación de la Espiritualidad en Contextos de Salud. Estudio descriptivo y transversal, realizado con 80 pacientes internados por lesión renal en hemodiálisis. Datos obtenidos de diciembre de 2011 a enero de 2012, por medio de una escala autoaplicable de evaluación de la espiritualidad. De los 80 pacientes, $93,75 \%$ presentaban alguna creencia religiosa. Cuanto a las expresiones de espiritualidad, 57,5\% estaban de acuerdo con la variable "Mis creencias espirituales/religiosas dan sentido a mi vida"; $70 \%$, con "Mi fe y creencia me dan fuerza en los momentos difíciles"; $48,8 \%$, con "Miro el futuro con esperanza"; $21,3 \%$, "Siento que mi vida se cambió para mejor"; e 63,7\%, con "He aprendido a valorar las pequeñas cosas de la vida". Se constató que el espiritual/religioso ejerce grande influencia en la salud del paciente con lesión renal en hemodiálisis, evidenciándose la importancia de profesionales preparados para proceder con esta temática. DESCRIPTORES: Espiritualidad; Insuficiencia Renal Crónica; Diálisis Renal; Enfermería.. 


\section{INTRODUÇÃO}

A Doença Renal Crônica (DRC) caracteriza-se pela perda progressiva e irreversível da função renal, resultando na inabilidade dos rins em exercer suas funções básicas de excreção e manutenção da homeostase. Tem incidência em torno de 2 a $5 \%$ dos pacientes hospitalizados, podendo ser influenciada por fatores como sepse, hipovolemia, uso de aminoglicosídeos, insuficiência cardíaca e radiocontrastes ${ }^{(1)}$.

Constitui-se hoje um importante problema de saúde pública no Brasil, visto que a prevalência de pacientes mantidos em programas crônicos de diálise dobrou nos últimos oito anos e, devido ao crescente número de indivíduos com lesão renal no país, este se tornou o terceiro maior mercado de hemodiálise do mundo. A mortalidade pela doença renal é 10 a 20 vezes maior que a da população geral, sendo a doença cardiovascular a causa mais comum de óbito ${ }^{(2)}$.

O paciente portador de enfermidades crônicas constantemente se depara com situações cuja complexidade vai além do quadro clínico provocado pela doença; lida com o sofrimento advindo da experiência de momentos difíceis, de grande rejeição, culpa e lutas, na tentativa diária de conviver harmoniosamente com sua condição de saúde ${ }^{(3)}$. A DRC impõe ao indivíduo uma série de mudanças e novas perspectivas de vida, incluindo o uso contínuo de medicação, o enfrentamento de dependência de outras pessoas e de aparelhos para adaptação à nova realidade e, na maioria dos casos, a dependência financeira.

A convivência com a enfermidade e o doloroso tratamento gera conflitos existenciais que podem provocarangústiaespiritualque, porsuavez, agrava os sintomas físicos e emocionais e a capacidade para enfrentar a doença. Dentre as diversas formas de fortalecimento do paciente, destacase a busca de sua espiritualidade, no intuito de sustentar suas dificuldades e adaptações ${ }^{(4)}$. Estudo realizado acerca da espiritualidade de pacientes portadores de DRC revelou que ela exerce grande importância na convivência com a doença e que a compreensão das experiências espirituais vividas pelos pacientes ajuda na promoção de um cuidado espiritual adequado ${ }^{(5)}$.

Enfatiza-se que o cuidado holístico na prática de enfermagem deve considerar as necessidades físicas, emocionais, sociais, econômicas e espirituais dos pacientes, sua resposta à enfermidade e o efeito desta sobre a capacidade para satisfazer sua necessidade de cuidado próprio ${ }^{(6)}$. Torna-se, portanto, necessária a compreensão das crenças religiosas e das diversas formas de expressar a religiosidade/ espiritualidade.

Baseando-se na teoria das Necessidades Humanas Básicas de Wanda Horta, percebeu-se a necessidade de resgate do cuidado holístico, no qual a espiritualidade aponta para uma importante dimensão na qualidade de vida do paciente, integrante das necessidades física e psicológica, fatores predominantes no cuidado ao paciente renal $^{(3)}$.

Espera-se que o estudo possa contribuir para a prática de enfermeiros que atuam na nefrologia, visto que a espiritualidade pode apresentar-se como forma de equilíbrio e força de vida, favorecendo o ajustamento da doença em pacientes com DRC, com resultados que poderão trazer subsídios para melhorar a prática assistencial em enfermagem.

Frente ao apresentado, foi objetivo deste estudo avaliar a espiritualidade de pacientes em hemodiálise, fundamentada na Escala de Avaliação da Espiritualidade em Contextos de Saúde.

\section{MÉTODO}

Trata-se de um estudo descritivo, transversal, com abordagem quantitativa. A pesquisa foi realizada em uma unidade de hemodiálise, de um serviço de nefrologia de um hospital terciário de grande porte, conveniado ao Sistema Único de Saúde (SUS), na cidade de Fortaleza, Ceará, Brasil.

A população do estudo correspondeu a 80 pacientes que estavam internados com lesão renal, realizando hemodiálise no período da coleta e atenderam aos critérios de inclusão estabelecidos no estudo: pacientes com idade mínima de 18 anos, de ambos os sexos, em condição clínica para responder ao instrumento autoaplicável e que aceitaram participar do estudo mediante assinatura do Termo de Consentimento Livre e Esclarecido.

A coleta de dados foi realizada no período de dezembro de 2011 a janeiro de 2012. Para o levantamento dos dados foram utilizados dois instrumentos: inicialmente, aplicou-se um questionário com perguntas relacionadas às características sociodemográficas dos pacientes; em seguida, aplicou-se a Escala de Avaliação da Espiritualidade em Contextos de Saúde, construída e validada ${ }^{(7)}$ no Brasil. 
A escala é constituída por cinco itens: 1. As minhas crenças espirituais/religiosas dão sentido à minha vida; 2 . A minha fé e crenças dão-me forças nos momentos difíceis; 3. Vejo o futuro com esperança; 4 . Sinto que a minha vida mudou pra melhor; 5 . Aprendi a dar valor às pequenas coisas da vida. As respostas são do tipo Likert, dadas numa escala de quatro alternativas, entre "não concordo" e "concordo plenamente".

Os dados de todas as variáveis foram analisados por meio de suas frequências absolutas e relativas com a utilização do programa estatístico Epilnfo, versão 3.5.1. Os resultados foram apresentados de forma descritiva e através de tabelas e figuras, a fim de possibilitar uma melhor visualização.

Ressalta-se que a pesquisa foi aprovada pelo Comitê de Ética e Pesquisa da referida instituição, sendo aprovado pelo número 201006/11, em concordância com as determinações da Resolução $n^{\circ}$ 196/96 do Conselho Nacional de Saúde ${ }^{(8)}$. A cada um dos pacientes foi realizada uma explicação prévia sobre os propósitos do trabalho e a garantia de seu anonimato.

\section{RESULTADOS}

Os participantes do estudo foram caracterizados em relação às variáveis: sexo, faixa etária, estado civil, escolaridade, profissão e naturalidade, apresentadas na Tabela 1.

Conforme observado, houve predomínio de participantes do sexo feminino (51,3\%), de faixa etária acima de 50 anos (45\%) e casados (53,75\%).
De acordo com a escolaridade percebeu-se a mesma quantidade de participantes que tinham o $1^{\circ}$ e o $2^{\circ}$ Graus incompletos, com $18(22,5 \%)$ cada. No que se refere à profissão, $19(23,75 \%)$ eram aposentados e donas de casa. Quanto à procedência, $53(66,25 \%)$ eram dos municípios do estado do Ceará; 17 (21,25\%) da capital; e dez $(12,5 \%)$ de outros estados.

No que diz respeito às crenças religiosas/ espiritualidade, observou-se que $56 \quad(70 \%)$ afirmaram ser católicos; 16 (20\%), evangélicos; um $(1,25)$ espírita; dois $(2,5 \%)$ relataram ter outras crenças; e cinco (6,25\%), referiram não ter religião. Analisando-se a distribuição de expressões de espiritualidade e crenças pessoais segundo pacientes com lesão renal, evidenciadas na Figura 1 , verificou-se que a maioria dos pacientes, 46 $(57,5 \%)$ estava plenamente de acordo quanto à expressão "As minhas crenças espirituais/ religiosas dão sentido à minha vida", enquanto 28 (35\%) responderam que concordavam bastante, quatro $(5 \%)$ concordavam um pouco e dois $(2,5 \%)$ não concordavam. Ao analisarmos as respostas dadas à expressão referente "A minha fé e crenças dão forças nos momentos difíceis" observou-se que $56(70 \%)$ pacientes estavam plenamente de acordo, 19 (23,8\%) concordavam bastante, quatro $(5 \%)$ concordavam um pouco e um $(1,3 \%)$ não concordava.

Em relação à expressão "Vejo o futuro com esperança", 39 (48,8\%) expressaram que estavam plenamente de acordo, 25 (31,3\%) concordavam bastante, $11(13,8 \%)$ concordavam um pouco e cinco $(6,3 \%)$ não concordavam. Quando se refere

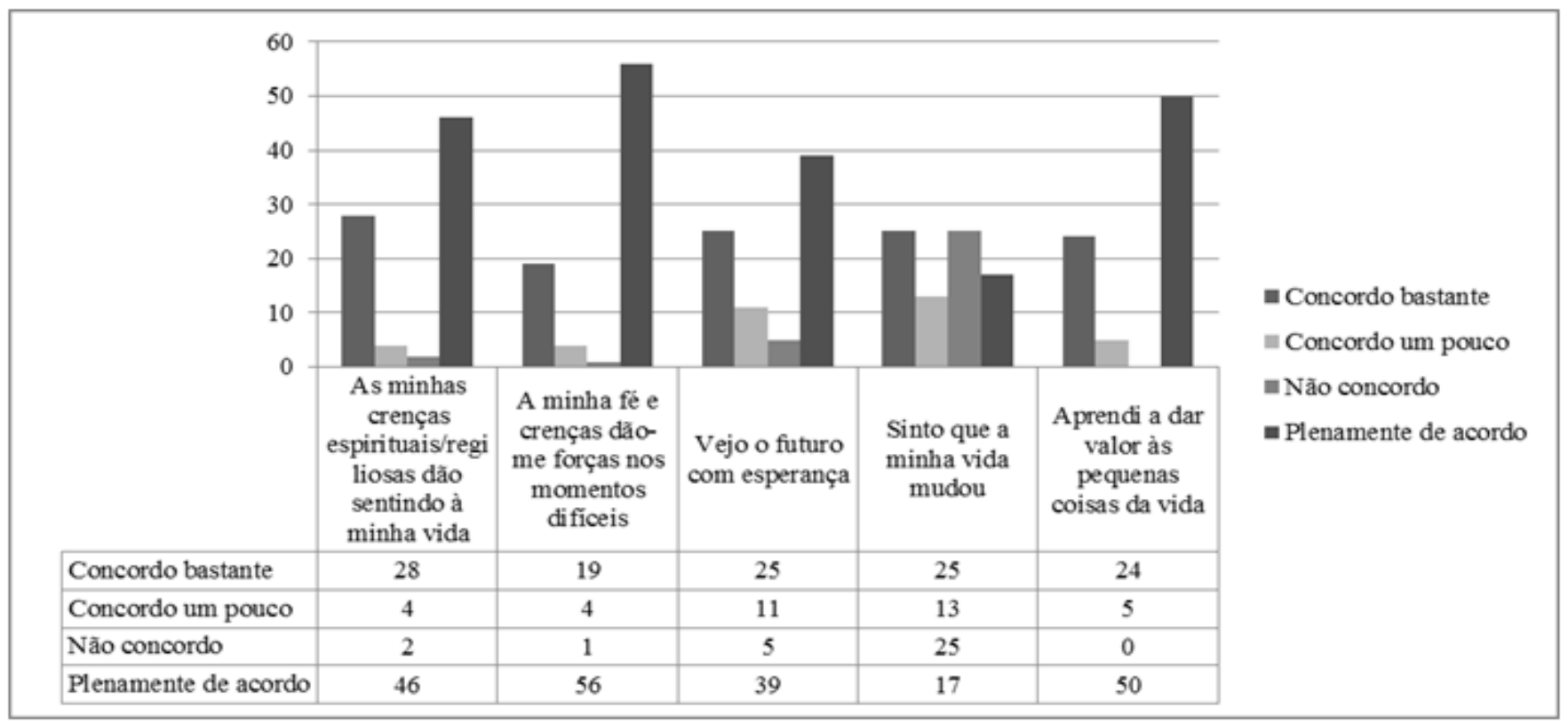

Figura 1 - Distribuição das expressões referentes às crenças religiosas pessoais/espiritualidade, segundo os participantes do estudo. Fortaleza, CE, Brasil, 2012 
Tabela 1 - Distribuição dos participantes do estudo segundo as variáveis sociodemográficas. Fortaleza, CE, Brasil, 2012

\begin{tabular}{|c|c|c|}
\hline Variáveis & $\mathbf{N}$ & $\%$ \\
\hline \multicolumn{3}{|l|}{ Sexo } \\
\hline Feminino & 41 & 51,25 \\
\hline Masculino & 39 & 48,75 \\
\hline \multicolumn{3}{|l|}{ Faixa etária } \\
\hline $18-19$ anos & 4 & 5 \\
\hline $20-29$ anos & 13 & 16,25 \\
\hline $30-39$ anos & 13 & 16,25 \\
\hline $40-29$ anos & 14 & 17,5 \\
\hline Acima de 50 anos & 36 & 45 \\
\hline \multicolumn{3}{|l|}{ Estado civil } \\
\hline Casado & 43 & 53,75 \\
\hline Divorciado & 10 & 12,5 \\
\hline Solteiro & 21 & 26,25 \\
\hline União estável & 4 & 5 \\
\hline Viúvo & 2 & 2,5 \\
\hline \multicolumn{3}{|l|}{ Escolaridade } \\
\hline Analfabeto & 13 & 16,25 \\
\hline $1^{\circ}$ Grau incompleto & 18 & 22,5 \\
\hline $1^{\circ}$ Grau completo & 14 & 17,5 \\
\hline $2^{\circ}$ Grau incompleto & 18 & 22,5 \\
\hline $2^{\circ}$ Grau completo & 13 & 16,25 \\
\hline Nível superior incompleto & 2 & 2,5 \\
\hline Nível superior completo & 2 & 2,5 \\
\hline \multicolumn{3}{|l|}{ Profissão } \\
\hline Aposentado & 19 & 23,75 \\
\hline Dona de casa & 19 & 23,75 \\
\hline Mecânico & 3 & 3,75 \\
\hline Agricultor & 9 & 11,25 \\
\hline Estudante & 7 & 9,75 \\
\hline Outros & 23 & 28,75 \\
\hline \multicolumn{3}{|l|}{ Naturalidade } \\
\hline Capital & 17 & 21,25 \\
\hline Municípios do estado do Ceará & 53 & 66,25 \\
\hline Municípios de outros estados & 10 & 12,5 \\
\hline \multicolumn{3}{|l|}{ Crenças religiosas/Espiritualidade } \\
\hline Católico & 56 & 70 \\
\hline Evangélico & 16 & 20 \\
\hline Espírita & 1 & 1,25 \\
\hline Outras & 2 & 2,5 \\
\hline Nenhuma & 5 & 6,25 \\
\hline
\end{tabular}

\section{DISCUSSÃO}

Apesar de outras pesquisas evidenciarem o predomínio do sexo masculino entre o público adulto com DRC, os resultados obtidos neste estudo destacaram o sexo feminino como o mais prevalente. Este achado pode estar agregado à dificuldade no serviço público de saúde que, embora dê atenção à saúde da mulher, a população encontra entraves no sistema. Ressaltam-se, também, as várias atribuições que a mulher acumula para si, como maternidade, trabalhadoras do lar e fora do lar, que podem trazer consequências negativas à saúde, a falta do autocuidado e o surgimento de doenças crônicas que levam ao internamento em instituições hospitalares ${ }^{(9)}$.

A faixa etária predominante mostrou-se de acordo com o apontado na literatura, que afirma que a idade média dos pacientes que desenvolvem a DRC é aos 60 anos $^{(10)}$. Quanto ao estado civil, a maioria dos participantes era casada, o que proporciona maior segurança para pacientes por fazer parte de uma estrutura familiar oficializada e estruturada, oferecendo um fortalecimento emocional e espiritual ao paciente, influenciando na sua recuperação e posterior adaptação. Autores ressaltam que a maioria dos pacientes conta com o apoio dos familiares e que muitas vezes, desde o início da doença, os amigos se afastam totalmente ficando apenas o amparo familiar - geralmente esposas e filhos ${ }^{(11)}$.

Verificou-se um baixo nível de escolaridade em grande parte dos participantes, o que pode favorecer uma adaptação ineficaz ao tratamento e trazer influências na continuidade do tratamento e adesão às orientações recebidas. Autores relatam que a maioria das pessoas em hemodiálise apresenta baixo índice de escolaridade, condição que contribui para limitação do acesso e da compreensão dos cuidados com a saúde ${ }^{(12)}$. 
Referindo-se à profissão dos participantes, observou-se o predomínio de aposentados e donas de casa. Provavelmente a situação é causada pela idade dos pacientes entrevistados, o que favorece a preocupação dos familiares quanto à possibilidade de vir a perder ou reduzir muitas vezes a única fonte de renda da família. Quanto ao fato da maioria das mulheres ser formada por donas de casa, leva-se em conta a idade e o estilo de vida de cuidarem da casa e dos filhos.

No tocante à naturalidade, a maioria era do interior do estado. O deslocamento para a capital é uma realidade comum em grande parte da população de renais, o que demanda disponibilidade de tempo para se submeter à hemodiálise. Isto gera mais uma mudança em sua vida com grande repercussão nas atividades cotidianas $^{(13)}$.

Observou-se o predomínio da religião católica. O Brasil destaca-se como um país em que a maioria da população considera-se cristã. A influência da religiosidade tem demonstrado grande influência sobre a saúde física, definindo-se como possível fator de prevenção ao desenvolvimento de doenças na população e eventual redução de óbito ou impacto de diversas doenças. As evidências têm se direcionado de forma mais firme e consistente para o cenário de prevenção. Estudos tentando avaliar qual a relação entre redução de mortalidade e prática religiosas têm enfatizado o possível incentivo que essas práticas oferecem a hábitos de vida saudáveis, suporte social, menores taxas de estresse e depressão ${ }^{(4)}$.

Acerca da distribuição das expressões referentes à espiritualidade/crenças pessoais, constatou-se a maioria dos participantes do estudo estava plenamente de acordo com a expressão "As minhas crenças espirituais/ religiosas dão sentido à minha vida". As crenças espirituais e religiosas são benéficas para o ser humano, pois ativam o ser espiritual que este é. Pesquisa constatou existir correlação forte entre a atitude religiosa e a realização existencial, sendo que a atitude religiosa pode ser vista como a expressão ou o ato de possuir crenças em algo superior, de forma a encontrar sentido na vida, na relação sujeito-mundo(14).

Outra expressão que os pacientes referiram estar plenamente de acordo foi "A minha fé e crença dão-me força nos momentos difíceis". Pode-se inferir o quão importante é a fé para o ser humano. Autores evidenciam que a fé é uma opção de tratamento, fortalecendo a coragem e a paciência para o enfrentamento das situações difíceis ${ }^{(3)}$.

Estudo recente constatou que pacientes que consideram a religião/espiritualidade como algo importante ou muito importante em suas vidas apresentam um alto escore de coping religioso/ espiritual, o que fortalece as estratégias utilizadas pelos mesmos como forma de enfrentar a condição de saúde ${ }^{(15)}$. Autores corroboram com esses achados ao afirmarem que ter uma "boa relação" com Deus ou com um ser superior fortalece a resiliência do indivíduo, ou seja, a capacidade do ser humano de responder às demandas da vida cotidiana de forma positiva, apesar das adversidades ao longo do seu ciclo vital de desenvolvimento, resultando na combinação positiva entre os atributos do indivíduo e seu âmbito familiar, social e cultural(16).

Referindo-se à expressão "Vejo o futuro com esperança", a maioria relatou concordar bastante. Em pesquisa nacional que buscou avaliar a correlação entre a esperança e a espiritualidade, verificou-se que há correlação positiva, de moderada magnitude. Dessa forma, como a esperança é algo essencial para o ser humano, e tem o poder de ajudar a enfrentar situações difíceis, impulsionando-o no seu cotidiano de forma benéfica, a religiosidade e a espiritualidade podem estar associadas à esperança de vida, pois a dimensão espiritual é descrita como relevante na atribuição de significados à vida e como um recurso de esperança no enfrentamento de doenças $^{(14)}$.

Observou-se uma divergência nas respostas referentes à expressão "Sinto que minha vida mudou para melhor". A doença renal pode estar associada a outras enfermidades que deixam os pacientes com uma sensação generalizada de mal estar, desinteresse pelas atividades cotidianas, medo, frustração e insegurança, dificuldades funcionais, afetivas, comportamentais e sociais; levando a um processo de adaptação à realidade em que se encontra e também devido à própria incerteza gerada pela internação hospitalar ${ }^{(13)}$. Assim, o portador de DRC precisa ser visualizado em sua totalidade, respeitando-se a sua individualidade e história de vida cotidiana, reintegração no contexto familiar e social, reconstrução da cidadania, enfim, que seja dado um enfoque biopsicossocial.

A fé traz conforto e bem estar ao indivíduo, influenciando positivamente sua maneira de viver. Em estudo que objetivou avaliar a qualidade de vida de pacientes em hemodiálise, a fé esteve mais fortemente associada ao sentimento de 
mudanças positivas, focando especialmente a espiritualidade, a religião e as crenças pessoais como fatores importantes que devem ser considerados e respeitados no momento da assistência prestada ${ }^{(17)}$.

A expressão "Aprendi a dar valor às pequenas coisas da vida" foi bastante assinalada positivamente entre os participantes. As situações difíceis produzem no ser humano experiências de superação fantásticas que o levam a adquirir novas experiências de vida, profundas mudanças de perspectivas e de comportamento. Isto influencia diretamente na valorização das pequenas coisas da vida, como a convivência com a família, a individualidade, a própria saúde, as amizades e, principalmente, o relacionamento com Deus e com o próximo ${ }^{(18)}$.

Destaca-se que a internação hospitalar produz uma reflexão sobre a vida e verdadeiros sentimentos e valores próprios de cada indivíduo. A forma como se experimenta o sofrimento é pessoal e há distintas possibilidades para lidar com esta experiência, seja retirando do sofrimento lições que poderão levar ao crescimento pessoal e a recuperar os valores principais da vida, seja se revoltando e caindo em desespero. Este desespero não tem apenas um significado trágico, porque às vezes ele é necessário para que a pessoa resignifique sua vida. A crise pode ser o pilar mestre de uma mudança ${ }^{(5)}$.

Destaca-se na literatura quão importante é o enfermeiro atento aos sinais apresentados pelos pacientes com o diagnóstico de enfermagem/ espiritualidade prejudicada, para que possa elucidar as informações e os significados embutidos nesta experiência, bem como avaliar a interveniência de aspectos da espiritualidade nas condições clínicas desses pacientes ${ }^{(6,19)}$.

\section{CONCLUSÃO}

Os resultados deste estudo revelaram que o aspecto espiritual/religioso do paciente com doença renal possui grande relevância para sua saúde por exercer influência direta na sua qualidade de vida, sendo de extrema importância que o enfermeiro considere a opinião do paciente sobre sua espiritualidade, como também deve utilizar instrumentos para avaliação do sofrimento e atividade espiritual.

Buscou-se, também, ampliar as possibilidades para a identificação de diagnósticos relacionados à dimensão espiritual, levando à assistência de enfermagem para a perspectiva holística, acarretando grande número de benefícios para o paciente e seus familiares.

Ressalta-se, portanto, a importância de uma equipe de saúde multidisciplinar preparada para essa abordagem de espiritualidade e religiosidade na vida de pacientes, e que saibam lidar com tais sentimentos e comportamentos. Infelizmente o Brasil ainda não tem a espiritualidade como temática na grade curricular dos cursos relativos à saúde.

Espera-se que esse estudo possa contribuir para despertar o interesse na temática, visto que se vive em um país religioso, com culturas diversas e comprovadamente uma população que necessita de cuidados específicos e seguros em relação à sua espiritualidade, tendo a enfermagem uma formação profissional holística com o compromisso de promover assistência supridora de necessidades básicas em toda a dimensão do ser humano.

\section{REFERÊNCIAS}

1. Pinto $C F$, Watanabe $M$, Fonseca $C D$, Ogata $C l$, Vattimo MFF. A sepse como causa de lesão renal aguda: modelo experimental. Rev. Esc. Enferm. USP. 2012; 46(esp):86-90.

2. Cesarino CB, Borges PP, Ribeiro RCHM, Ribeiro DF, Kusumota L. Avaliação do risco cardiovascular de pacientes renais crônicos segundo critérios de Framingham. Acta Paul. Enferm. 2013; 26(1):101-7.

3. Lucchetti G, Almeida LGC, Granero AL. Espiritualidade no paciente em diálise: o nefrologista deve abordar? J Bras Nefrol [Internet] 2010; 32(1) [acesso em 12 fev 2012]. http://dx.doi.org/10.1590/ S0101-28002010000100020

4. Santos CCE, Gomes AMT, Oliveira DC, Pontes APM, Santos EI, Costa CPM. Diálogos entre espiritualidade e enfermagem: uma revisão integrativa da literatura. Cogitare enferm. 2013; 18(2):372-8.

5. Chaves ECL, Carvalho EC, Dantas RAS, Terra FS, Nogueira DP, Souza L. Validação da escala de espiritualidade de Pinto e Pais Ribeiro em pacientes com insuficiência renal crônica em hemodiálise. Rev Enferm UFPE on line [Internet] 2010; 4(2) [acesso em 25 fev 2012]. Disponível: http://www.revista.ufpe. br/revistaenfermagem/index.php/revista/article/ download/890/1322

6. Cortez EA. Influência da religiosidade e espiritualidade na saúde: reflexões para o cuidado de enfermagem. Online Braz J Nurs [Internet] 2012; 11(2) [acesso em 07 jan 2013]. Disponível: http://dx.doi. org/10.5935/1676-4285.2012S001. 
7. Pinto C, Pais-Ribeiro JL. Construção de uma escala de avaliação da espiritualidade em contextos de saúde. Arq. med. [Internet] 2007; 21(2) [acesso em 10 fev 2012]. Disponível: http://www.scielo.oces.mctes.pt/pdf/am/ v21n2/v21n2a02.pdf

8. Ministério da Saúde (BR). Conselho Nacional de Saúde. Dispõe sobre diretrizes e normas regulamentadoras de pesquisas envolvendo seres humanos. Resolução n. 196, de 10 de outubro de 1996. Brasília; 1996.

9. Cravo CDL, Miranzi SSC, Iwamoto HH, Júnior JLS. Epidemiological profile of patients of a university hospital on hemodialysis. Cienc Cuid Saúde. 2011; 10(1):110-15.

10. Frazão CMFQ, Ramos VP, Lira ALBC. Qualidade de vida de pacientes submetidos a hemodiálise. Rev. enferm. UERJ. 2011; 19(4):577-82.

11. Grasselli CSM, Chaves ECL, Simão TP, Botelho PB, Silva RR. Avaliação da qualidade de vida dos pacientes submetidos à hemodiálise. Rev Bras Clin Med. 2012; 10(6):503-7.

12. Costa FAP, Cavalcante MCV, Lamy ZC, Filho NS. Cotidiano de portadores de doença renal crônica: Percepção sobre a doença. Rev. Med. Minas Gerais. 2009;19(4 supl 2):12-7. 13. Santos RP, Rocha DLB, Koch VHK. Componentes clínico-epidemiológicos de crianças e adolescentes em hemodiálise. Cogitare enferm. 2014; 19(2):361-7.

14. Ottaviani AC, Souza EN, Drago NC, Mendiondo MSZ, Pavarini SCI, Orlandi FS. Esperança e espiritualidade de pacientes renais crônicos em hemodiálise: estudo correlacional. Rev. Latino-Am. Enfermagem. 2014; 22(2):248-54.

15. Valcanti CC, Chaves ECL, Mesquita AC, Nogueira DA, Carvalho EC. Coping religioso/espiritual em pessoas com doença renal crônica em tratamento hemodialítico. 2012; 46(4):838-45.

16. Martínez BB, Custódio RP. Relationship between mental health and spiritual wellbeing among hemodialysis patients: a correlation study. São Paulo Med. J. 2014; 132(1):23-7.

17. Rusa SG, Peripato GI, Pavarini SCI, Inouye K, Zazzetta MS, Orlandi FS. Qualidade de vida/ espiritualidade, religião e crenças pessoais de adultos e idosos renais crônicos em hemodiálise. Rev. LatinoAm. Enfermagem. 2014; 22(6):911-7.

18. Rocha NS, Fleck MPA. Avaliação de qualidade de vida e importância dada a espiritualidade/ religiosidade/crenças pessoais (SRPB) em adultos com e sem problemas crônicos de saúde. Rev Psiq Clín. $2011 ; 38(1): 19-23$.
19. Pedrão RB, Beresin R. O enfermeiro frente à questão da espiritualidade. Einstein. 2010; 8(1pt1):86-91. 